Rabaska

Revue d'ethnologie de l'Amérique française

\title{
Bibliographie du père Anselme Chiasson
}

\section{Ronald Labelle}

Volume 2, 2004

URI : https://id.erudit.org/iderudit/201651ar

DOI : https://doi.org/10.7202/201651ar

Aller au sommaire du numéro

Éditeur(s)

Société québécoise d'ethnologie

ISSN

1703-7433 (imprimé)

1916-7350 (numérique)

Découvrir la revue

Citer ce document

Labelle, R. (2004). Bibliographie du père Anselme Chiasson. Rabaska, 2,

149-155. https://doi.org/10.7202/201651ar d'utilisation que vous pouvez consulter en ligne.

https://apropos.erudit.org/fr/usagers/politique-dutilisation/ 


\section{Bibliographie du père Anselme Chiasson}

1. LiVReS

a) Ouvrages personnels

1961. Chéticamp : histoire et traditions acadiennes. Préface de Luc Lacourcière, Moncton, Éditions des Aboiteaux, 1961, 317 p. ; $4^{\mathrm{e}}$ édition avec supplément, 1961-1989, Richibouctou, Éditions Babineau, 1990, $333 \mathrm{p}$.

1969. Les Légendes des Îles-de-la-Madeleine. Moncton, Éditions des Aboiteaux, 1969, 123 p. ; $2^{\mathrm{e} e ́ d i t i o n, ~ M o n c t o n, ~ E ́ d i t i o n s ~ d ' A c a d i e, ~ 1976, ~}$ $132 \mathrm{p}$.

1981. Les Îles-de-la-Madeleine : vie matérielle et sociale de l'empremier. Montréal, Leméac, « Connaissance », 1981, 269 p.

1983. Tout le long de ces côtes : chansons folkloriques des îles-de-laMadeleine. Mont Saint-Hilaire (Qc), Chant de mon pays, 1983, 64 p.

1990. Paroisse Saint-François-d'Assise d'Ottawa, 1890-1990. Ottawa, publié par la paroisse, 1990, $235 \mathrm{p}$.

1991. Le Diable Frigolet et 24 autres contes des Îles-de-la-Madeleine. Moncton, Éditions d'Acadie, 1991, 224 p.

1994. Contes de Chéticamp. Moncton, Éditions d'Acadie, 1994, 192 p.

1995. Le Nain jaune et autres contes des Iles-de-la-Madeleine. Moncton, Éditions d'Acadie, 1995, $130 \mathrm{p}$.

1996. The Seven-Headed Beast and Other Acadian Tales from Cape-Breton Island. Traduit par Rosie Aucoin Grace, Wreck Cove (N.-É.), Breton Books, 1996, 190 p.

1998. Chéticamp : History and Acadian Traditions. Traduction par Jean Doris LeBlanc, Wreck Cove (N.-É.), Breton Books, 1998, 275 p.

1999. Anna Malenfant, Gloire de l'Acadie et du Canada, cantatrice acadienne. Moncton, Éditions d'Acadie, 1999, 130 p. 


\section{b) Ouvrages écrits en collaboration}

1942. Chansons d'Acadie, $1^{\text {ere }}$ série. En collaboration avec Daniel Boudreau, Montréal, La Réparation, 1943, 32 p.

1945. Chansons d'Acadie, $2^{e}$ série. En collaboration avec Daniel Boudreau, Montréal, La Réparation, 1945, 53 p.

1946. Chansons d'Acadie, $3^{e}$ série. En collaboration avec Daniel Boudreau, Montréal, La Réparation, 1946, 52 p.

1966. Les Acadiens d'aujourd'hui. En collaboration avec René Beaudry, Rapport de recherche préparé pour la Commission royale d'enquête sur le bilinguisme et le biculturalisme, Ottawa, 1966, 2 volumes.

1967. L'Île de Shippagan. Anecdotes, tours et légendes. En collaboration avec Francis Savoie, Moncton, Éditions des Aboiteaux, 1967, 95 p.

1969. Chansons d'Acadie, $4^{e}$ série. En collaboration avec Daniel Boudreau, Moncton, Éditions des Aboiteaux, 1972, 57 p.

1971. L'Histoire du Nouveau-Brunswick. En collaboration avec Émery LeBlanc et Jean Daigle, Toronto, Gage, 1971, 96 p.

1976. Petit Manuel d'histoire d'Acadie. En collaboration avec Jean Daigle, Clarence d'Entremont et Léon Thériault, Moncton, Librairie acadienne, 1976, $38 \mathrm{p}$.

1979. Chansons d'Acadie, $5^{e}$ série. En collaboration avec Daniel Boudreau, Moncton, Éditions des Aboiteaux, 1979, 60 p.

1981. L'Île de Lamèque, anecdotes, tours et légendes. Moncton, Éditions d'Acadie, 1981, 93 p.

1985. L'Histoire des tapis " hookés " de Chéticamp et de leurs artisans. En collaboration avec Annie-Rose Deveau, Yarmouth, N.-É., Éditions Lescarbot, 1985, 180 p. illustrées en couleurs.

1986. Sainte-Anne de Kent, 1886-1986. En collaboration avec Arthur Poirier, Sainte-Anne, Éditions Chockpish, 1986, 159 p.

1988. The History of Cheticamp Hooked Rugs and their Artisans. Traduit par Marcel LeBlanc, Yarmouth, N.-É., Éditions Lescarbot, 1988, $183 \mathrm{p}$.

1988. Camille Lefebvre, c.s.c. En collaboration avec les pères Clément Cormier et Maurice Chamard, Montréal, Fides, 1988, 230 p.

1993. Ristigouche, Centenaire des Capucins, 1894-1994. En collaboration avec le père Albert Landry, publié par les pères Capucins de Ristigouche, $1993,175 \mathrm{p}$. 
1993. Ristigouche Centenary of the Capucins, 1894-1994. Traduit par Gerald Kelly, 1993.

1996. Chansons d'Acadie. En collaboration avec Daniel Boudreau, Moncton, Centre d'études acadiennes, $1996,6^{\mathrm{e}}$ série, 65 p. ; $7^{\mathrm{e}}$ série, 57 p. ; $8^{\mathrm{e}}$ série, 65 p. ; $9^{\mathrm{e}}$ série, 69 p. ; $10^{\mathrm{e}}$ série, $76 \mathrm{p}$; $111^{\mathrm{e}}$ série, $81 \mathrm{p}$.

1996. Chéticamp, Mémoires d'Anselme Boudreau. Moncton, Éditions des Aboiteaux, 1996, réécrit et annoté par le père Anselme Chiasson, $228 \mathrm{p}$.

2002. Chansons d'Acadie, séries 1 à 4. Nouvelle édition, en collaboration avec Daniel Boudreau, Moncton, Centre d'études acadiennes, 2002, $216 \mathrm{p}$.

\section{Articles}

1961. "Monographie paroissiale », Les Cahiers de la Société historique acadienne, Moncton, premier cahier, 1961, p. 11-17.

1965. « La Famille des Malenfant aux Maritimes », Les Cahiers de la Société historique acadienne, Moncton, vol. 1, no 8, 1965, p. 19-20.

1966. « Voyage historique des Acadiens en Europe », Les Cahiers de la Société historique acadienne, Moncton, vol. 2, $\mathrm{n}^{\circ}$ 2, 1966, p. 45-52.

1969. «Les Vieilles Maisons acadiennes », Les Cahiers de la Société historique acadienne, Moncton, vol. 3, n ${ }^{\circ}$ 5, 1969, p. 183-187.

1969. " Notre voyage en Louisiane », Les Cahiers de la Société historique acadienne, Moncton, vol. 3, n 3, 1969, p. 123-128.

1970. "Les dix années de la Société historique acadienne », Les Cahiers de la Société historique acadienne, Moncton, vol. $3, \mathrm{n}^{\circ} 8,1970$, p. 328330.

1970. «Placide Gaudet (sa vie, son ouvre) », Les Cahiers de la Société historique acadienne, Moncton, vol. $3, \mathrm{n}^{\circ} 3$, septembre 1970 , p. 120 128.

1971. « Placide Gaudet (1850-1930) », Les Cahiers de la Société historique acadienne, Moncton, vol. 4, $\mathrm{n}^{\circ} 1,1971$, p. 6-23.

1972. "Le Centre d'études acadiennes de l'Université de Moncton », The French in New England, Acadia and Quebec, New England - Atlantic Provinces - Quebec Center, University of Maine in Orono, 1972, p. 43-48. 
1973. "Centre d'études acadiennes », Les Cahiers de la Société historique acadienne, Moncton, vol. 4, n 8, 1973, p. 313-324.

1974. « Biographie du père René Patrice », Dictionnaire biographique du Canada, Québec, Presses de l'Université Laval, 1974, vol. 3, p. 597598.

1975. "L'Inventaire général des sources documentaires sur les Acadiens ", Les Cahiers de la Société historique acadienne, Moncton, vol. $8, \mathrm{n}^{\circ} 2$, mai 1975, p. 151-153.

1976. « Le Centre d'études acadiennes de l'Université de Moncton et son folklore ", Revue de l'Université Laurentienne, vol. 8, $\mathrm{n}^{\circ} 2,1976, \mathrm{p}$. 115-121.

1977. «Monographie paroissiale », La Revue de la Société historique du Madawaska, le Brayon, vol. 5, no 4, 1977, p. 8-11.

1977. « Cantiques acadiens de 1755 », Les Cahiers de la Société historique acadienne, Moncton, vol. 8, $\mathrm{n}^{\circ}$ 1, 1977, p. 35-41.

1978. « Le Clergé et le réveil acadien (1864-1960)», Les Cahiers de la Société historique acadienne, Moncton, vol. 11, n¹, février 1978, p. 29-46.

1978. "Luc Lacourcière et l'Acadie ", dans Mélanges en l'honneur de Luc Lacourcière, sous la direction de Jean-Claude Dupont, Montréal, Leméac, 1978, p. 11-12.

1979. «L'Évolution historique du folklore en Acadie depuis un siècle », Les Cahiers de la Société historique acadienne, Moncton, vol. 10, $\mathrm{n}^{\circ} 4$, 1979 , p. 170-175.

1980. « Les Traditions et la littérature orale en Acadie », dans Les Acadiens des Maritimes : études thématiques, sous la direction de Jean Daigle, Moncton, Centre d'études acadiennes, 1980, p. 521-556.

1981. « Geneviève Massignon (1921-1966) », Les Cahiers de la Société historique acadienne, Moncton, vol. 12, $\mathrm{n}^{\circ} 3,1981$, p. 118-123.

1981. « Monographie paroissiale et histoire orale ", dans Histoire orale/Oral History, sous la direction de Ronald Labelle, Moncton, 1981, p. 5-10.

1984. «L'Encan des morts », Bulletin d'histoire et de généalogie/La Société Saint-Pierre, vol. 1, n 3 , 1984, p. 3.

1986. « Nos chansons de Chéticamp - leur renommée », Bulletin d'histoire et de généalogie/La Société Saint-Pierre, vol. 3, n² 2, 1986, p. 7-9.

1987. « Culture et activités culturelles », Encyclopédie du Canada, 1987, p. $1-8$. 
1990. "An Acadian Story : Les trois enfants brûlés ", Cape Breton's Magazine, $\mathrm{n}^{\circ}$ 55, 1990, p. 6-68.

1991. « La Messe du revenant/Mass of the Ghost », Cape Breton's Magazine, $n^{0} 57,1991$, p. 67-68.

1992. « L'Enfant à la bonne compagnie », Cape Breton's Magazine, $\mathrm{n}^{\circ}$ 61, 1992, p. 31-40.

1993. Chiasson, Anselme, Charlotte Cormier, Donald Deschênes et Ronald Labelle, "Le Folklore acadien », dans L'Acadie des Maritimes : études thématiques des débuts à nos jours, sous la direction de Jean Daigle, Moncton, Chaire d'études acadiennes, 1993, p. 649-705.

1993. «L'Eau qui rajeunit/The Fountain of Youth », Cape Breton's Magazine, $n^{0} 64,1993$, p. 59-66.

1995. Chiasson, Anselme, Charlotte Cormier, Donald Deschênes et Ronald Labelle, "Acadian Folklore», dans Acadia of the Maritimes : Thematic Studies from the Beginning to the Present, sous la direction de Jean Daigle, Moncton, Chaire d'études acadiennes, 1995, p. 625-678.

1999. «Les Pains bénits », Les Cahiers de la Société historique acadienne, vol. $30, n^{\circ} 3,1999$, p. 165-168.

\section{3. Écrits sur le père Anselme Chiasson}

1976. « Hommage au père Anselme Chiasson », Parallèle 48, sept.-oct. 1976, p. 8.

1981. Andrée Nolet, Étude des régionalismes lexicaux tirés de la chronique acadienne: "Le Coin à Piquine », thèse (M.A.), Université Laval, Québec, 1981.

1982. Ronald Labelle et Lauraine Léger (dir.), En r'montant la tradition, hommage au père Anselme Chiasson, Moncton, Éditions d'Acadie, $1982,254 \mathrm{p}$.

1993. Charlotte Cormier et Donald Deschênes, « Anselme Chiasson et Germain Lemieux, deux folkloristes en milieu minoritaire ", dans L'Euvre de Germain Lemieux, s.j. Bilan de l'ethnologie en Ontario français, sous la direction de Jean-Pierre Pichette, Sudbury, Prise de parole et Centre franco-ontarien de folklore, 1993, p. 147-158.

1993. Donald Deschênes, « Portrait d'auteur : Anselme Chiasson », Francophonies d'Amérique, no 3, Ottawa, 1993, p. 147-154. 
1999. Charlotte Cormier, « La Réception de Chansons d'Acadie (1942-1955) d'Anselme Chiasson. La correspondance : analyse lexicale d'une construction identitaire ", Les Cahiers des la Société historique acadienne, Moncton, vol. 30, $\mathrm{n}^{\circ} 2$ (juin 1999), p. 76-84.

2003. Ronald Labelle, " Portrait du père Anselme Chiasson », Bulletin Mnémo, hiver 2003, p. 2-3.

2004. Paul-André Savoie, « Personnalité acadienne : père Anselme Chiasson, capucin, historien et ethnologue ", L'Aboiteau. Bulletin de liaison de la Fédération acadienne du Québec, hiver 2004, p. 6-9.

\section{HoNNEUR, dÉCORATIONS, PRIX}

1961. Prix Champlain et Prix du Concours littéraire et scientifique, Ministère des Affaires culturelles, Québec, pour la publication de Chéticamp, histoire et traditions acadiennes.

1976. Membre de l'Ordre du Canada.

1976. Doctorat honoris causa en histoire, Université de Moncton.

1978. Doctorat honoris causa en histoire, Université Sainte-Anne, Pointede-l'Église, Nouvelle-Écosse.

1979. Membre de l'Ordre des Francophones d'Amérique et récipiendaire $d u$ Grand Prix du 3 juillet 1608, Conseil de la langue française du Québec.

1980. Nommé Folkloriste de l'année par l'Association canadienne d'ethnologie et de folklore.

1984. Décoré des Palmes académiques de France.

1991. Reçu Associé de la Compagnie des Cent associés francophones d'Amérique.

1995. Prix d'excellence Pascal-Poirier (littérature), ministère des Affaires culturelles, Nouveau-Brunswick.

1995. Prix de Grand-Pré, du gouvernement de la Nouvelle-Écosse pour son livre Contes de Chéticamp.

1996. Médaille Marius Barbeau, Association canadienne d'ethnologie et de folklore.

1997. Élu membre à vie, Association canadienne d'ethnologie et de folklore.

1999. Chevalier de l'Ordre national du Mérite, de la République française, Médaille remise par le Président Chirac lors du Sommet de la francophonie à Moncton. 
1999. Médaille Léger-Comeau de la Société nationale de l'Acadie.

1999. Plaque d'honneur du Conseil culturel acadien, Nouvelle-Écosse, « pour sa contribution littéraire à la préservation de notre mémoire collective $"$.

2001. Prix Hommage du festival acadien Éloizes pour l'envergure de son œuvre et sa contribution à la culture acadienne.

2004. Officier de l'Ordre du Canada. 